\title{
NMR metabolomics reveals effects of Cryptosporidium infections on host cell metabolome
}

\author{
Christopher N. Miller ${ }^{1}$, Charalampos G. Panagos 2,5 , William R. T. Mosedale ${ }^{1}$, Martin Kváćc,4, Mark J. Howard ${ }^{2,6}$ \\ and Anastasios D. Tsaousis ${ }^{1 *}$ [D
}

\begin{abstract}
Background: Cryptosporidium is an important gut microbe whose contributions towards infant and immunocompromise patient mortality rates are steadily increasing. Over the last decade, we have seen the development of various tools and methods for studying Cryptosporidium infection and its interactions with their hosts. One area that is sorely overlooked is the effect infection has on host metabolic processes.

Results: Using a ${ }^{1} \mathrm{H}$ nuclear magnetic resonance approach to metabolomics, we have explored the nature of the mouse gut metabolome as well as providing the first insight into the metabolome of an infected cell line. Statistical analysis and predictive modelling demonstrated new understandings of the effects of a Cryptosporidium infection, while verifying the presence of known metabolic changes. Of note is the potential contribution of host derived taurine to the diarrhoeal aspects of the disease previously attributed to a solely parasite-based alteration of the gut environment, in addition to other metabolites involved with host cell catabolism.
\end{abstract}

Conclusion: This approach will spearhead our understanding of the Cryptosporidium-host metabolic exchange and provide novel targets for tackling this deadly parasite.

Keywords: Cryptosporidiosis, NMR, Metabolomics, COLO-680N, Taurine

\section{Background}

Cryptosporidiosis is a disease characterised by prolonged episodes of intense diarrhoea and is the second largest cause of diarrheal disease and diarrhoea-associated deaths in infants across Africa and South Asia [1-4]. The aetiological agents of this disease are the apicomplexan parasites belonging to the Cryptosporidium genus. Cryptosporidiosis is also amongst one of the most common diseases of immunocompromised individuals, particularly HIV positive patients who are at $75-100 \%$ risk of contracting the disease during their lifetime $[4,5]$. Nearly 20 Cryptosporidium species and genotypes are responsible for causing the disease in humans; although two

\footnotetext{
*Correspondence: A.Tsaousis@kent.ac.uk

1 Laboratory of Molecular \& Evolutionary Parasitology, RAPID Group,

School of Biosciences, University of Kent, Canterbury, UK

Full list of author information is available at the end of the article
}

species in particular, Cryptosporidium hominis and $C$. parvum, are most likely to be found in infected patients [2, 4, 6-9]. Infection occurs when an individual ingests the oocysts of the parasite, often by drinking a contaminated water source. Water treatment options are limited to filtering or boiling, which are generally not possible at an industrial scale and UV treatment, which is both expensive and rarely in place prior to outbreaks. Failing this, treatment is typically rehydration, although one drug has been shown to be effective, the broad spectrum anti-parasitic nitazoxanide [10]. The drug is far from ideal, however, and displays a range of undesirable side effects including cytotoxicity and nausea, as well as being limited to use in cases where the patients are immunocompetent [11-14].

Until recently, a significant barrier to research into cryptosporidiosis has been the absence of a combined long-term in vivo culturing system and comprehensive 
model of host parasite interactions in addition to a heavy reliance on antibody based detection both in the scientific and the medical field [1, 3, 15-19]. Recent papers have attempted to rectify this by proposing improved or entirely novel techniques for culturing the parasite ex vivo in cell cultures, using the cultured cancer cells as host cells $[20,21]$. A recent study identified that infection of COLO-680N cell cultures produced a longer term and higher production volume culture of the parasite compared to previously existing in vitro cultures $[22,23]$. These advances have allowed higher in depth microscopy-based studies and even promise to provide a solution to developing a genetic engineering platform for the parasite. However, beyond microscopy and localisation studies [24], the knowledgebase of the host parasite interaction remains largely undeveloped $[3,13,14$, $21,25]$. One area lacking study is metabolomics. The study of parasite metabolomics is becoming increasingly important as the search for preventative treatments and cures becomes increasingly specific [26]. However, before metabolomics can be used to satisfying effect, the tools must first be properly established and demonstrated.

To our knowledge, only two peer-reviewed publications have explored the concept of the infection metabolome, one on mice and the other on human faecal samples [27, 28]. The findings of each paper demonstrated a clear relationship between the infection and metabolic changes. Although working on different organisms and sampling sites, each study identified the hexadecanoic acid as a significant component of these changes. Other changes noticed included a decrease in the relative abundance of amino acids in infected mice faeces, although an increase was previously in humans [27]. This was explained to be most likely due to the inherent variation between the different host species metabolomes, as highlighted by Saric et al. in 2008 [29]. However, this highlights a pressing need for further and wider reaching studies into the metabolome of Cryptosporidium infections. One approach would be to increase the variety of tools available, in addition to the gas chromatography-mass spectrometry (GC-MS) used in those papers [27-29].

Currently, many metabolomics studies utilise a GCMS approach, with great success, however ${ }^{1} \mathrm{H}$ nuclear magnetic resonance (NMR) metabolomics can be used as an additional or alternative powerful tool for metabolic screening. ${ }^{1} \mathrm{H}$ NMR is a simple method that allows for a comparatively lossless analysis of metabolites, with fewer steps between sample recovery and analysis than GC-MS, offering a huge advantage for studies involving time sensitive or limited resources restrictions, such field research [29-33]. This translates to a more reliable result in terms of quantification and immediate reproducibility. As such, NMR has already seen use in analysing the profile of Plasmodium falciparum, although the metabolome of the apicomplexan parasite, as is the case with the rest of the group, remains largely unexplored compared to similar studies of other organisms [34].

Here we investigated the host-parasite interactions, using a combination of microscopy and ${ }^{1} \mathrm{H}$ NMR approaches. We validated our methodology by comparing faecal profile results to the previously published studies, which used different methodologies, namely GC-MS $[27,28]$. Further experimentation utilised a recently published infectible culture, COLO-680N [22], to determine if any similarities or differences in response to infection could be determined.

\section{Results}

\section{Cell culture sample extractions}

Extrapolated NMR data from COLO-680N $(\mathrm{n}=38, C$. parvum Iowa $=12$, control $=12, C$. hominis $=7, C$. parvum Weru=7) metabolite extractions, demonstrated clear differences between the metabolomes of each individual strain/species of Cryptosporidium infection (Fig. 1a). Differences could be observed between creatine, creatine phosphate, taurine and lactate spectra before the application of any analytical methods (Fig. 1b-d). Analysis using the Chenomx NMR Suite version 8.2 [35, 36] produced a list of 161 total compounds of varying concentrations across samples (Additional file 1: Figure S1). The partial least squares discriminant analysis (PLS-DA) generated, produced ample separation of the Cryptosporidium-infected and uninfected cultures in multiple experiments (Fig. 2a, c). Furthermore, the separation of the individual infection groups suggests that differences between both Cryptosporidium species and within individual strains of C. parvum, may elicit different metabolic responses in cell cultures. It is important to note that all data points obtained from the 38 individual samples were input into the calculations, as any outliers should be visible via the resulting PLS-DA plots and pre-emptive pruning of the data was deemed to be unreliable given the lack of pre-existing information on what should/shouldn't be expected. The loading scores plot of the PLS-DA showed several compounds contributed heavily to the separations between groups, such as lactate, several fatty acid derivatives and taurine (Fig. 2b).

All metabolites identified in this manner were input into an online tool (MetaboAnalyst 3.0) producing a graph detailing which metabolic pathways were influenced by infection (Additional file 2: Figure S2) [37]. This approach identified several pathways, including the biosynthesis of various amino acids, as well as ketones and CoA (Additional file 2: Figure S2b-f). Within these pathways, metabolites were highlighted that were identified via the PLS-DA as contributing reliably towards 


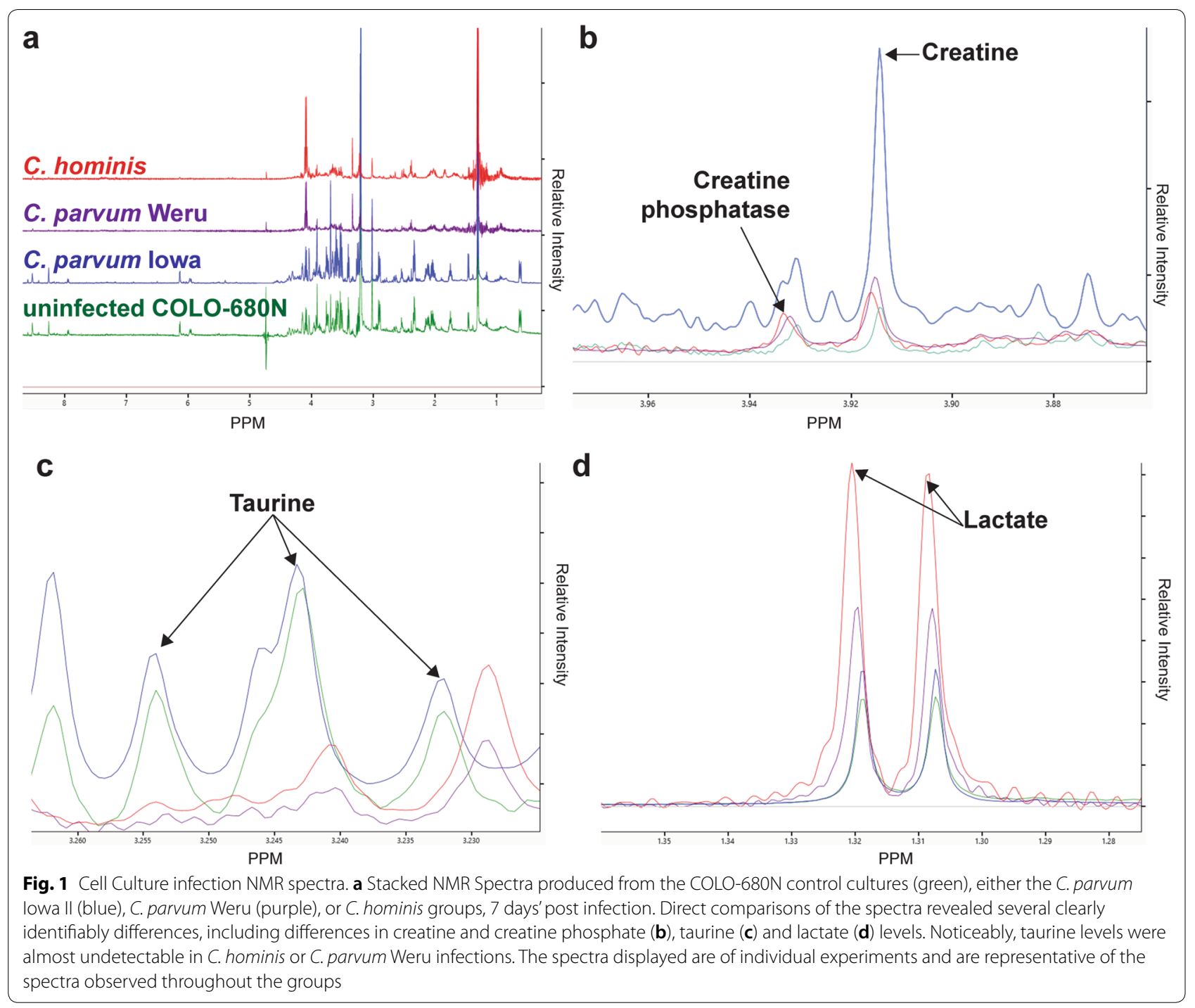

differences between groups. Full compound names are available in Additional file 3: Figure S3.

\section{Mice faecal sample extractions}

Faecal samples from infected and uninfected mice were smeared onto microscope slides and stained with an aniline-carbol-methyl violet method [38], allowing the detection of $C$. parvum oocysts and thus validation of successful infections (Additional file 4: Figure S4). Samples from both control and infected mice were taken at 10 days post infection, while monitoring and counting the number of oocysts. The spectra produced by the NMR showed clear distinctions between the infected and uninfected mice, as well as distinctions between the different strains of infections (Fig. 3a). Though 18 individual experiments were used to produce this data, the validity and reliability of each was confirmed by performing a further nine technical replicate NMR scans. Several metabolites were readily distinguishable prior to the metabolomics analyses, including indicators of phosphorylation; taurine (Fig. 3b), creatine and creatine phosphate (Fig. 3c) and lactate (Fig. 3d). Processing the data from the mice guts $(n=18$, six per infection) via the Chenomx NMR Suite version 8.2 platform produced a list of 151 compounds that were extrapolated from the spectra (Additional file 5: Figure S5). Statistical analysis of the data, with freely available Microsoft Excel Add-in "multi-base 2015", by PLS-DA determined some separation of the three conditions, (uninfected control, $C$. parvum Iowa II and C. parvum Weru infections), whilst maintaining group cohesion (Fig. 4a). The loading values of the variable compound contributions (Fig. 4b), suggest 


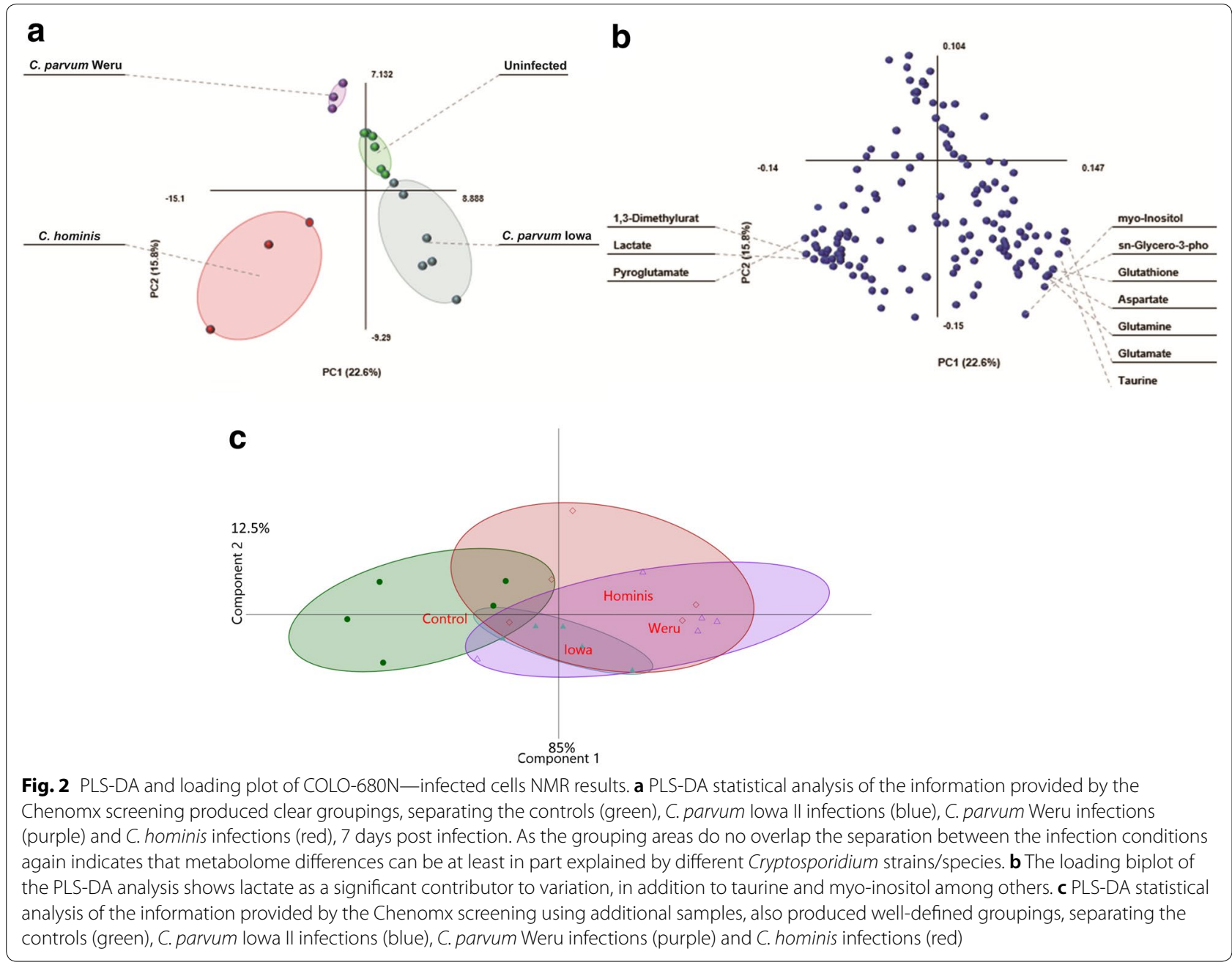

certain metabolites were more significant to the separation of the groups than others. The presence of L-alanine and valine, two common amino acids, agrees with the previous literature and 2-oxoisocaproate is a component of the valine/leucine/isoleucine biosynthetic pathways reports $[27,28]$.

MetaboAnalyst 3.0 based analysis of the metabolites proposed that several amino acid biosynthesis pathways could be altered during an infection, such as the glycine, valine and taurine pathways. In addition, the mice infections displayed possible changes to other metabolic pathways (Additional file 6: Figure S6a) as those pathways furthest from the $\mathrm{x}, \mathrm{y}$ axis intercept, representing both the overall completeness of the pathways and number of contributing detected metabolites respectively. As with Additional file 2: Figure S2a-g, the pathways identified in the manner, and the compounds discovered by the NMR demonstrated that infections caused changes in at least the valine (Additional file 6: Figure S6c), glycine (Additional file 6: Figure S6d) and taurine amino (Additional file 6: Figure S6e) acid biosynthetic pathways, in addition to several sugar pathways (Additional file 6: Figure S6b, f, g). As before, full compound names are available in Additional file 3: Figure S3.

\section{Comparison of mice faecal and COLO-680N metabolome changes}

MetaboAnalyst data from Additional file 2: Figure S2 and Additional file 6: Figure S6, demonstrate that a number of altered pathways are shared between the mice (faecal) and cell culture metabolites, particularly taurine and amino acid metabolic pathways. Glycine synthesis was also shown to be affected to a large degree. Comparing the data from the mouse and cell culture responses directly revealed many metabolite levels responded similarly to infection regardless of host (Fig. 5). 


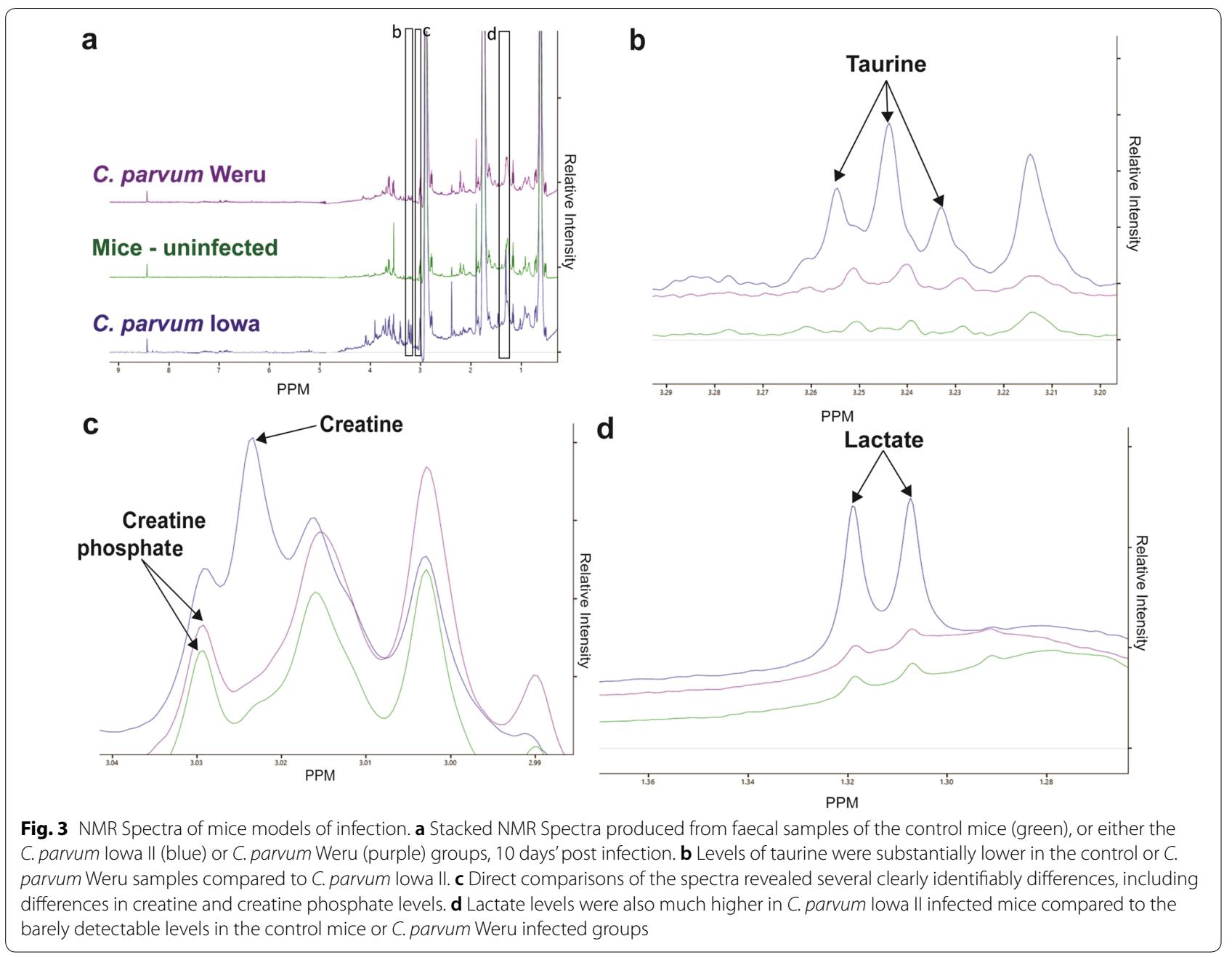

\section{Discussion}

Previous studies recently demonstrated the successful long-term propagation of C. parvum in COLO-680N cell culture [22]. The ability of the cell culture to maintain the parasite for up to 8 weeks [22, 23] along with the presence of organelles around the parasite (e.g. feeder organelle) [21], implied a metabolic association between the parasite and the host. To investigate this, we have used ${ }^{1} \mathrm{H}$ NMR to explore the metabolomics of the infection.

Solution-state ${ }^{1} \mathrm{H}$ NMR offers a practical approach to metabolomics that is especially useful where sample volume sizes are particularly small [32, 33, 39]. Although GC-MS holds an advantage for detecting low-levels of metabolites with unique mass signatures, to determine the change in metabolite quantities, NMR provides a viable alternative [29-34]. Initial analysis of our data showed a clear distinction between the metabolic fingerprints of infected and uninfected samples, even between infections of different strains of the parasite to some extent; with PLS-DA producing distinct groups of metabolite profiles, correlating to uninfected and infected samples (Fig. 2a, c). This may in-part be explained as the manifestation of the biochemical differences between the species which contribute to their observed species specificity.

Of importance is the degree to which these results, both from the in vitro and in vivo, agree with the previous literature. As a monolayered, simplistic culturing system, it should not be expected that the results would be a perfect mimicry of an in vivo experiment, although their similarity to other studies should indicate that the COLO-680N culture is a pragmatically sufficient model for infection in more generalised studies such as this one. Our study also demonstrates that metabolic compounds L-alanine, isoleucine and succinic acid (succinate) were detected as contributors to the variance between the sample conditions that indicated infection. Moreover, even though valine was not detected in the uninfected controls, it was visible in the infected samples and in agreement with previous studies [27, 28]. 


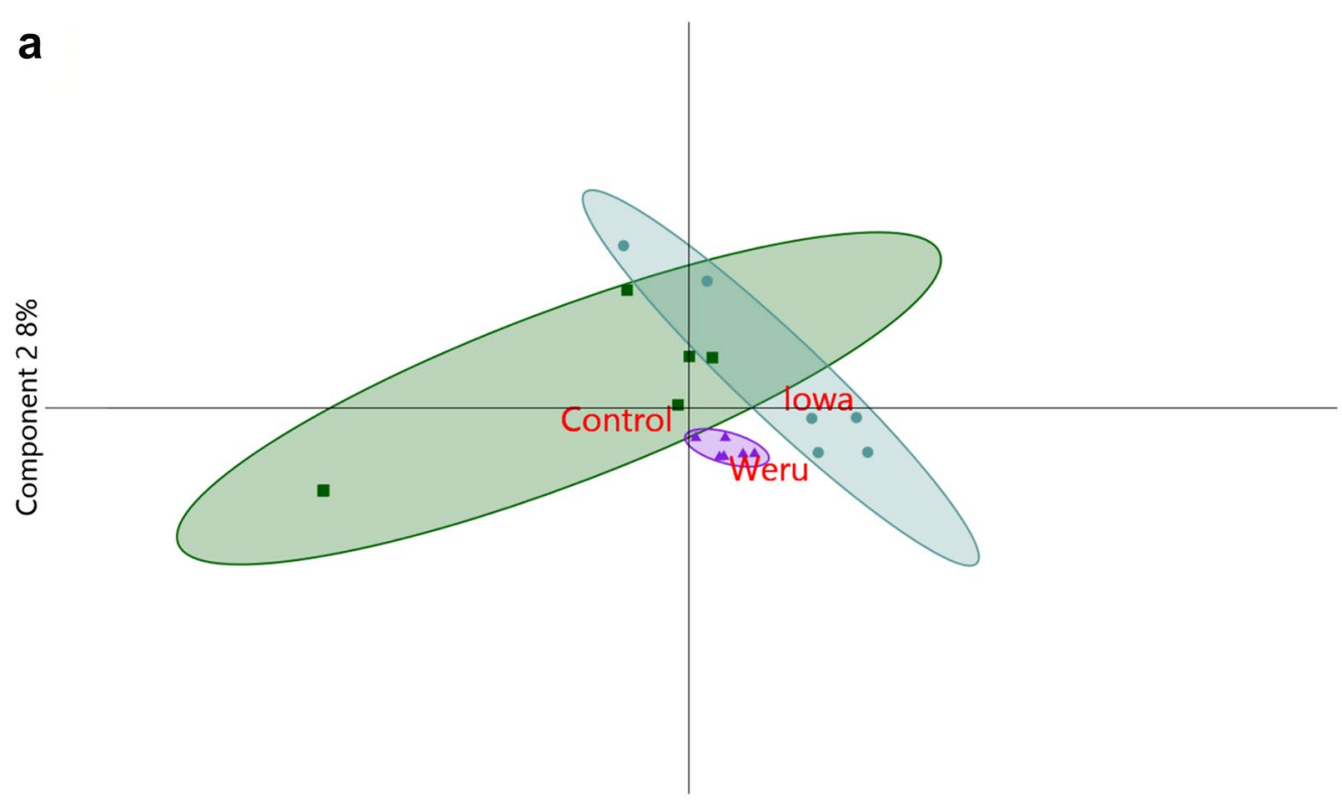

Component $191 \%$

b

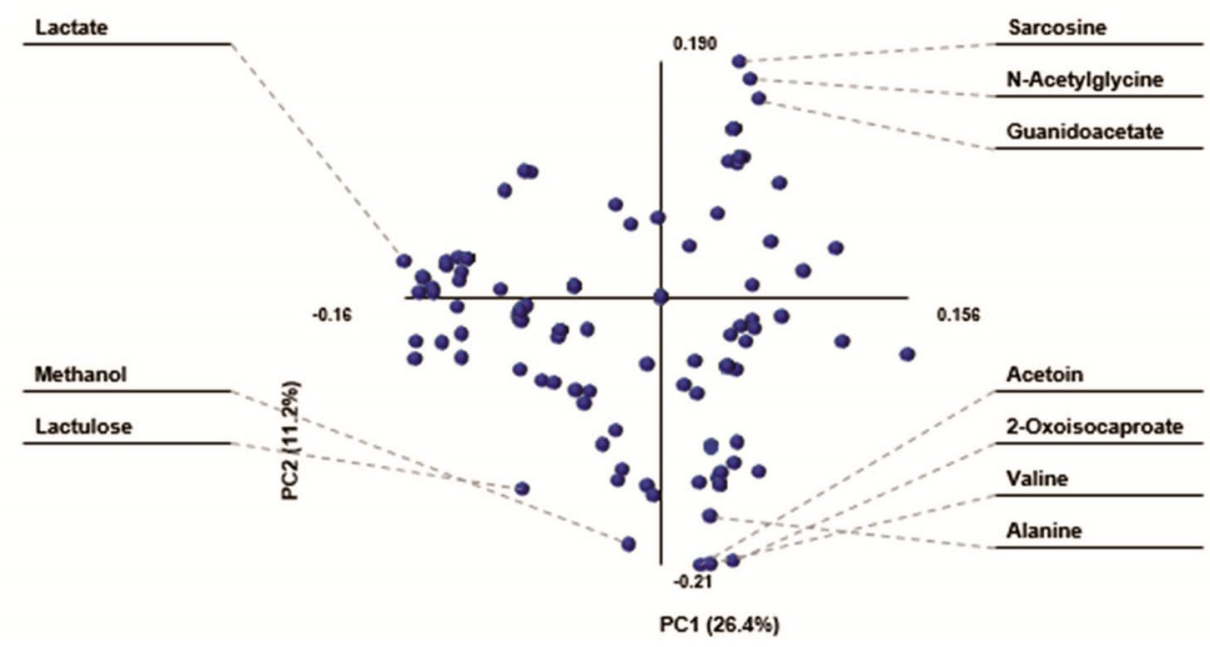

Fig. 4 PLS-DA and loading plot of mice model NMR results. a PLS-DA statistical analysis of the information provided by the Chenomx screening produced clear groupings, separating the controls (green), C. parvum lowa II infections (blue) and C. parvum Weru infections (purple), 10 days post infection. As the grouping areas, indicated by the areas highlighted, do overlap, it can be said that the separation between the infection conditions represent some differences in the metabolome, which correspond to the C. parvum strain. $\mathbf{b}$ The loading biplot of the PLS-DA analysis shows many of the compounds identified by Chenomx contributed towards the separation and groupings. Those on the outer most edges, for example alanine, sarcosine, lactate and lactulose, had some of the greatest influence on the amount of separation as determined by the PLS-DA

The MetaboAnalyst data revealed several pathways were potentially influenced by infection, including several that showed changes in both the mice and cell culture experiments, such as amino acid and CoA biosynthesis. Support for these findings is observed via the biosynthesis pathways for alanine and glycine that were highlighted previously in GC-MS studies as being potentially influenced by infection $[27,28]$. It is, however, important to note that these findings are based entirely on the presence/absence or relative changes in abundance of the noted metabolites and therefore cannot account for their specific origin. For example, it is not possible to distinguish between an increase in metabolite level due to damage of a pathway, compared 
a

\begin{tabular}{c|c|c|c|c}
\hline \multicolumn{5}{|c|}{ Shared changes in C. parvum lowa Il infections } \\
\hline & Mouse & Cells & Pathways & Function \\
\hline 1,3-Dihydroxyacetone & & & Glycolysis & Energy supply \\
\hline 3-Hydroxy-3-methylglutarate & & & Ketogensis & Ros scavenging \\
\hline Anserine & & & Carnosine synthesis & Numerous \\
\hline Asparagine & & Numerous & Cell surface signalling \\
\hline Aspartate & & AA Synthesis & Numerous \\
\hline Fucose & & & N-linked glycosylation & Enective tissue \\
\hline Glycine & & Purine synthesis & Numerous \\
\hline Glycylproline & & & Collagen synthesis & Getogensisis \\
\hline Isoleucine & & & Numerous & Negulation \\
\hline N-Acetylaspartate & & & Waste & Earcinogen \\
\hline N-Acetylornithine & & & Epigenetics & Energy supply \\
\hline N-Nitrosodimethylamine & & & Kumerous, CoA synthesis & Ros \\
\hline N6-Acetyllysine & & & Numerous & Osmoregulation \\
\hline Pantothenate & & & Sugar synthesis & Cytoskeleton and nuclear pore formation \\
\hline Syringate & & & Nolysaccharide synthesis & Lipid formation \\
\hline Taurine & & & Cell signalling andmitochondrial quality control \\
\hline UDP-N-Acetylglucosamine & & & &
\end{tabular}

b

\begin{tabular}{c|c|c|c|c}
\hline \multicolumn{3}{|c|}{ Shared changes in C. parvum Weru infections } \\
\hline & Mouse & Cells & Pathways & Function \\
\hline 2-Hydroxybutyrate & & & Cysteine synthesis & Oxidative stress response \\
\hline Acetone & & & Ketosis & Energy supply \\
\hline Citrate & & & TCA & Energy supply \\
\hline Creatine & & Creatine synthesis & Metabolic regulation and methylation \\
\hline Formate & & & Folate cycle & Potentially Ketosis based energy supply \\
\hline Levulinate & & & Unknown & Potential parasite energy source \\
\hline Mannitol & & & Mannitol cycle (Non-mammalian) & Regulation of inflammation \\
\hline Methylguanidine & & Protein catabolism & Glutatmate storage \\
\hline Pyroglutamate & & & Glutathione cycle & Energy Supply \\
\hline Valine & & & CoA synthesis & \\
\hline
\end{tabular}

Fig. 5 Shared changes in metabolite levels between both cell cultures and mice. Those metabolites which showed a reliable contribution towards group separation (determined by positive PLS-DA values, displayed as green shaded cells in Additional file 1: Figure S1 and Additional file 5: S5) in both mice and cell culture experiments were recorded and their functions assigned. Those metabolites with direct or indirect involvement with mitochondria are labelled in red. The analysis was conducted for both the C. parvum lowa II (a) and C. parvum Weru (b) infection experiments. *N-nitrosodimethylamine is a known carcinogen and not naturally produced by any known human or mammalian cell line or any member of the cryptosporidia and may represent either a product of the gut microbiota, contamination or un-characterised spectra peak

to deliberate upregulation of a pathway, or even to the alterations of the gut microbiota. We hope to mitigate this by comparing the previous standards of these experiments, the mouse faecal samples, to the cell culture results.

As a parasite, Cryptosporidium is dependent on host derived biosynthetic pathways for survival. For example, C. parvum is incapable of producing most amino acids de-novo, instead relying heavily on the import of host metabolites via active channelling [40]. The biosynthetic pathway for glycine, threonine and serine was upregulated, in both cell culture and animal experimentations, with particularly high levels of glycine detected. Both $C$. parvum and $C$. hominis are incapable of manufacturing these amino acids de novo, instead relying on scavenging host serine and glycine, utilising serine and glycine hydroxymethyltransferases to convert one to the other when needed [40, 41]. The reliance on host amino acids could provide a novel method for combating the infection, based upon previous studies that identified other amino acid metabolic chains as potential targets [41, 42]. For example, glycine reuptake inhibitors (GRIs) that are often used in treating schizophrenia, could be utilised to partially starve the parasite of the metabolite.

In addition to the amino acid biosynthesis pathways, it is also apparent that taurine synthesis is also implicated in the metabolic profile of the disease as shown in the presented analyses; taurine has frequently been used in the past as an agent for inducing excystation for in vitro cultures as sodium taurocholate [43-46]. In the host, taurine has several roles, those relevant to the cell types involved include: cell membrane integrity, osmoregulation and adipose tissue regulation. Perhaps most notably, however, is the role taurine plays as a $\mathrm{pH}$ regulator in the host mitochondria. The addition of taurine is another piece of evidence indicating host-mitochondria are somehow directly involved in the progress of infection. Previous metabolomic studies of faecal samples from Cryptosporidium-infected patients revealed increased taurine concentrations, which was explained away as characteristic of a decline in gut absorption as a result villi malformation [47, 48]. However, malabsorption is not an applicable explanation in the infected COLO$680 \mathrm{~N}$ cell cultures, wherein there is no external source of 
the metabolite and thus is likely correlated to the infection metabolome. Increases were also observed in the abundance of adenosine derivatives (AMP, ADP and ATP); all showing an increased abundance in infected cells and mice in C. parvum Iowa II infections, along with a similar increase in creatine levels in C. parvum Weru infections. This further implicates the role of host mitochondria in the context of infection as each species and strain of parasite lacks creatine kinase, the only alternative source therefore being host creatine kinase which is often found in close association with mitochondria. Levels of pyruvate in C. hominis cell cultures and pantothenate in C. parvum Iowa II mouse infections suggest interactions with oxidative phosphorylation. This is of interest as the $C$. parvum genome contains a sequence for a potential pantothenate scavenging protein [49]. Moreover, the further increase in lactate levels detected in C. hominis cell cultures and C. parvum Iowa II mouse infected samples, compared to the controls, indicate a strong contribution from anaerobic pathways most likely from the host. This suggests that more ATP is being produced than the oxidative capacity of the host mitochondria alone can maintain, producing a net increase in lactate as the oxygen debt increases. This suggests either an atypical drain of cellular ATP or a decrease in host cell aerobic capacity. Similar observations have been made in other intracellular parasites, including the microsporidian Encephalitozoon cuniculi, in which the organism acquired specialised transporters to overcome its needs for ATP [50].

The findings above suggest that $C$. parvum and $C$. hominis infections are directly or indirectly inducing an increase in host mitochondrial activity. If factual, this would result in many oxygen free radicals being produced by the metabolic machinery. Consequently, cell(s) would respond with a matching increase in the synthesis of antioxidants such as taurine, which also sees increases during infection [51-53]. However, there also exists nonrelated rationale for the detected increase in taurine, for example as a diuretic, which should not be a surprise in cryptosporidiosis, which is characterised by excessive water loss. This role sees taurine maintaining levels of the ionised forms of magnesium and potassium within the cell, producing a diuretic effect that may contribute towards the characteristic water-loss [48, 54-56]. Furthermore, it has been found that taurine levels influence production of short chained fatty acid, another aspect of host biology theorised to be scavenged by C. parvum and C. hominis [56-58]. Previous studies which have identified a rise in taurine levels in cryptosporidiosis patients' stool, have dismissed the event as simply the result of the guts decrease in absorptive qualities. The presence of increased taurine in the in vitro samples, which lack external sources that could be responsible for a build-up, would appear to dispute this conclusion. It is our interpretation, therefore, that the intracellular role of taurine in this disease has been overlooked and that the pathophysiology of this disease is more complicated than currently understood, extending beyond villi degradation.

Lastly, these results provide a potential for determining infections via a possible comparative ${ }^{1} \mathrm{H}$ NMR of patient and reference biopsies. This would offer an alternative approach in the medical field, where current methods of diagnosis are reliant on multiple, separate, techniques to achieve the same result as NMR, with infections detected by laborious and often inaccurate microscopy in tandem with strain typing dependant on a successful PCR.

\section{Conclusion}

In conclusion, we have demonstrated for the first time that the use of ${ }^{1} \mathrm{H}$ NMR in the context of both medical and scientific applications is indispensable in the fight against cryptosporidiosis. With the application of a more user-friendly and reproducible approach of metabolomics, through the ${ }^{1} \mathrm{H}$ NMR methodology described in this paper, it will now be easier for the Cryptosporidium community to further explore the remaining aspects of the disease metabolome in patients' samples.

\section{Methods \\ Cryptosporidium}

Three isolates of Cryptosporidium were used in this study. The reference strain $C$. parvum Iowa II was obtained from Bunch Grass Farm in the United States, isolated from infected calves. The human isolate Cryptosporidium parvum Weru strain was originally isolated from an infected human patient and subsequently maintained by passing through SCID mice and supplied courtesy of Prof. Martin Kváč of the Institute of Parasitology Biology Centre CAS, Czech Republic. The final isolate used was the human isolate of $C$. hominis, supplied courtesy of Prof. Rachel Chalmers from the Cryptosporidium Reference Unit, Singleton Hospital of NHS Wales.

\section{Cell culture}

$75 \mathrm{~cm}^{2}$ monolayers of COLO-680N were infected and maintained as per the protocols outlined previously [22], using all three isolates of Cryptosporidium. In brief, for a typical infection, $4 \times 10^{6}$ oocysts were used to infect $75 \mathrm{~cm}^{2}$ cell culture flasks at between 70 and $80 \%$ confluency $\left(2 \times 10^{6}\right.$ cells $)$ giving a multiplicity of infection (MOI) of approximately 2 . Infected cells were incubated for 7 days and monitored daily for their infectivity [22, 23]. Prior to sample collection and metabolite extraction, the level of infection was monitored using Sporo-glo live staining under fluoresce microscopy [22, 24]. A control 
group was also established, following the same protocols as the infections, absent oocysts. Two separate experiments were executed using a minimum of five flasks per sample condition.

\section{Animals and infection}

Pregnant female BALB/c mice (Charles River, Germany) were housed in plastic cages with sterilized wood-chip bedding situated in IVC Air Handling Solutions (Techniplast, Italy) with high-efficiency particulate air (HEPA) filters and supplied with sterilized food and water ad libitum. For this study, 7 day old BALB/c mice from the same mother, habiting the same cage were infected at the Institute of Parasitology, Biology Centre CAS using pre-established protocols detailed in Meloni and Thompson, totalling five mice per condition [59]. Three separate conditions, totalling six animals each, were used, infecting with 100,000 oocysts of C. parvum Iowa II resuspended in $50 \mu$ l of PBS, 100,000 oocysts of the C. parvum Weru isolate resuspended in $50 \mu \mathrm{l}$ of PBS or a PBS control $(50 \mu \mathrm{l})$, given by oral gavage. The groups were kept physically separated and never allowed to interact. Infection was monitored and oocyst production was quantified from day-1 post-infection by aniline-carbol-methyl violet staining of faecal smears [60], RIDA ${ }^{\circledR}$ QUICK Cryptosporidium, supplied by R-Biopharm. At 10-days post-infection, the mice were euthanized by cervical dislocation and decapitation. Samples of the ileum were dissected from the mice, measured to the same size to ensure reproducibility. Cryptosporidium hominis was not used in the mice infection experiments as it has previously shown that this species cannot infect these animals [61].

\section{Sample preparation for NMR}

The following protocol was adapted from published and well-established metabolic extraction methods used for NMR-based untargeted analysis of cell extracts [62-65]. Samples collected from the mouse experiments were retrieved from the contents of the ileum and surrounding intestinal structure. A section of ileum approximately $5 \mathrm{~mm}$ in length was removed from the euthanised mouse by scalpel. A syringe containing $3 \mathrm{ml}$ of $100 \%$ ethanol at room temperature was the inserted into the removed ileum and the ethanol pushed through the ileum over a petri dish. The sample was then collected via pipette and stored in three $1.5 \mathrm{ml}$ tubes in $1 \mathrm{ml}$ aliquots.

Collected samples were then centrifuged for $3 \mathrm{~min}$ at $10,000 \times g$, the supernatant discarded, and the pellet weights recorded. The samples were then suspended by vortex in $2 \mathrm{ml}$ of $75 \%$ ethanol, pre-heated to $80{ }^{\circ} \mathrm{C}$, to immediately inhibit subsequent metabolic reactions, then transferred to a new tube and an additional five $\mathrm{ml}$ of $75 \%$ ethanol added.

Two microlitre of $2 \mathrm{~mm}$ diameter glass beads were added to the samples and agitated by vortex for $30 \mathrm{~s}$ before incubating the samples for $3 \mathrm{~min}$ at $80{ }^{\circ} \mathrm{C}$. The samples were vortexed for a further $30 \mathrm{~s}$ or until the sample was completely homogenised. Cell culture samples were collected by draining the media, adding $6 \mathrm{ml}$ of ethanol at $80{ }^{\circ} \mathrm{C}$ to the culture flask and scraping the cells off the surface by cell scraper, transferring the mixture of lysed cells into $15 \mathrm{ml}$ polyethylene tubes via a $10-\mathrm{ml}$ serological pipette.

The samples were then transferred into $2 \mathrm{ml}$ tubes, retaining the glass beads in $15 \mathrm{ml}$ conical tubes. The beads were washed with an additional two $\mathrm{ml}$ of $80{ }^{\circ} \mathrm{C}$, $75 \%$ ethanol and again the liquid was transferred into sterile $2 \mathrm{ml}$ tubes, retaining the glass beads in the tube.

Cell debris and general detritus were separated from the metabolite samples by centrifugation at $16,000 \times g$ for $10 \mathrm{~min}$ at room temperature and the resulting supernatant transferred to new, sterile $2 \mathrm{ml}$ microcentrifuge tubes and the remaining debris weighed for data normalisation. The samples were then dried via Rotorvac for $12 \mathrm{~h}$ or until completely desiccated, at $40{ }^{\circ} \mathrm{C}$, suspended in $330 \mu \mathrm{l}$ double distilled water and centrifuged at $2500 \times g$ for $10 \mathrm{~min}$. The supernatants were recombined into $\sim 1 \mathrm{ml}$ aliquots per original sample in sterile $1.5 \mathrm{ml}$ microcentrifuge tubes and frozen at $-20{ }^{\circ} \mathrm{C}$ until the day before NMR analysis. The sample tubes are subsequently placed into a freeze drier until completely desiccated, suspended in $1 \mathrm{ml}$ of deuterium oxide $\left({ }^{2} \mathrm{H}_{2} \mathrm{O}\right)$ and spiked with the sodium salt of the calibration and quantitation control compound: 3-(trimethylsilyl)-1-propanesulfonic acid (DSS), to a final concentration of $20 \mu \mathrm{M}$ and a tested $\mathrm{pH}$ of 7.5.

\section{NMR protocol and analysis}

Samples were analysed using a 4-channel Bruker Avance III 14.1 T NMR spectrometer $\left(600 \mathrm{MHz}{ }^{1} \mathrm{H}\right)$ equipped with a $5 \mathrm{~mm}$ QCI-F cryoprobe. For controls: six separate, uninfected $25 \mathrm{~cm}^{2}$ COLO-680N 100\% confluent monolayer cultures were analysed in addition to three uninfected $\mathrm{BALB} / \mathrm{c}$ mice. Infected samples consisted of six $25 \mathrm{~cm}^{2}$ COLO-680N 100\% confluent monolayers in addition to three $c$. parvum Iowa II infected BALB/c and three C. parvum Weru infected BALB/c mice. Onedimension NMR datasets were acquired with a pulse repetition rate of $5 \mathrm{~s}$ over 128 scans, preceded by eight equilibrating dummy scans and suppression of the residual Deuterium Oxide solvent (HDO) resonance using presaturation. This was repeated 5 times per sample to ensure the reliability of the spectra produced. Processed NMR spectrographic datasets were produced by Topspin 
3.2 and analysed using Chenomx NMR Suite version 8.2. Partial Least Squares Discriminant Analysis (PLS-DA) of the Chenomx data were generated with the freely available Microsoft Excel Add-in "multi-base 2015" by Numerical Dynamics, Japan ("Mutlibase for Microsoft Excel," 2015) and "Past3.x" by Øyvind Hammer, Natural History Museum, University of Oslo. Pathway predictions were produced by the MetaboAnalyst 3.0 web tool, using a hypergeometric test and relative-betweeness centrality (measure of centrality in a graph based on shortest paths) against Homo sapiens and Mus musculus databases for the tissue culture and mouse models respectively [37]. However, the analysis methods do contain limitations, relying largely on human interpretation of the initial results. As such it is not possible to determine whether a result is erroneous or simply unexpected and be able to prove the decision was free of bias, we elected to include all the findings from the analysis in this paper. In this case, the limitation is also a result of database comprehensiveness, whilst standards can be used in the lab to determine the accuracy of the dataset, it cannot detect compounds it has not been trained to recognise. Furthermore, as a result it is possible that such compounds may be misidentified, caffeine for example is highly unlikely to be present in the sample but in the spirit of intellectual integrity the results have not be edited or altered in any way other than those required for easier reading. Furthermore, compounds have been labelled based on their most likely source (human metabolome for COLO-680N samples, mouse metabolome for faecal (gut microbiome) samples or C. parvum if not found in either of the previous), whilst some (such as acetyl ornithine) may serve a purpose beyond "waste product" in other organisms, in this paper we do not consider potential outside sources [37]. On the other hand, as is the case with all new technologies and techniques, these limitations can only shrink with repeated use as more data becomes available to fill in the gaps.

\section{Additional files}

Additional file 1: Figure S1. COLO-680N Experiment Metabolites. All the metabolites identified by $1 \mathrm{H}$ NMR analysis in infected and uninfected cells were explored via PLS-DA statistical analysis and the resulting values of each individual metabolite recorded. The colour coded heat map represents the significance to which each individual metabolite contributed to the identity of the sample groups. Red indicates that a metabolite showed large amounts of variance within identically treated samples, yellow indicates that the amount of a metabolite varied little throughout all samples and green indicates that the metabolite was uniform within both control and infection groups but demonstrated a marked difference between them. Colour intensities were determined relatively from loading values, using the lowest negative value and highest positive value as the anchor points for red and green respectively, anchoring the mid-point yellow as 0 .
Additional file 2: Figure S2. Metabolic pathways detected in cell cultures' NMR samples. a Data analysed by MetaboAnalyst 3.0, utilising all compounds which displayed some degree of change as a result of infection, produced a graph of pathways most heavily impacted ( $x$ axis) and pathways containing the most amount of the given compounds (pathway impact: $y$-axis), with statistical significance of the predicted pathways increasing as the colour ranges from yellow (low) to red (high). Six pathways were chosen to be of particular interest by their position on the graph, with metabolites present in the experimental samples highlighted in red, including: glycine, serine and threonine metabolism (b), taurine and hypotaurine metabolism (c), Alanine, aspartate and glutamate metabolism ( $\mathbf{d})$, synthesis and degradation of ketones (e), pantothenate and CoA biosynthesis (f) and arginine and proline metabolism (g).

Additional file 3: Figure S3. Compound code key. KEGG ID to Compound name conversion table for use with Additional file 2: Figure S2 and Additional file 5: Figure S5.

Additional file 4: Figure S4. Staining of Cryptosporidium in faecal samples. Aniline-carbol-methyl violet stain of a faecal smear taken from a mouse in the infection group. The abundant presence of Cryptosporidium oocysts (arrows) indicates that the infection has been successful; and that the animal is producing oocysts. These samples were acquired at 7 days post-infection

Additional file 5: Figure S5. Mice Experiment Metabolites. All the metabolites identified by ${ }^{1} \mathrm{H}$ NMR analysis in infected and uninfected mice were explored via PLS-DA statistical analysis, the Principal Component values for each metabolite were then recorded. Metabolites that contributed towards variation within groupings are coded towards the red, whilst green represents metabolites that stayed relative unvaried within groups but demonstrated variation between groups and thus are of most interest. Yellow represents a general lack of variation between or within groups.

Additional file 6: Figure S6. Metabolic pathways detected in mouse model NMR samples. a Data analysed by MetaboAnalyst 3.0, utilising all compounds which displayed some degree of change as a result of infection, produced a graph of pathways most heavily impacted ( $x$ axis) and pathways containing the most amount of the given compounds (pathway impact: $y$-axis), with statistical significance of the predicted pathways increasing as the colour ranges from yellow (low) to red (high). Six pathways were chosen to be of particular interest by their position on the graph, with metabolites present in the experimental samples highlighted in red, including: $\mathbf{b}$ pentose and glucuronate interconversions, valine, $\mathbf{c}$ valine, leucine and isoleucine biosynthesis, $\mathbf{d}$ glycine serine and threonine metabolism, e taurine and hypotaurine metabolism, $\mathbf{f}$ galactose metabolism and $\mathbf{g}$ starch and sucrose metabolism.

\section{Abbreviations}

NMR: nuclear magnetic resonance; DSS: 3-(trimethylsilyl)-1-propanesulfonic acid, sodium salt; PLS-DA: principal component analysis; PLS-DA: partial least squares discriminant analysis; UV: ultraviolet; HIV: human immunodeficiency virus; GC-MS: gas chromatography-mass spectrometry; HDO: deuterium oxide; PCR: polymerase chain reaction; PBS: phosphate-buffered saline; EM: electron microscopy; SCID: severe combined immunodeficiency disease; ATP: adenosine triphosphate; AMP: adenosine monophosphate; ADP: adenosine diphosphate; CoA: coenzyme A; GRIs: glycine reuptake inhibitors.

\section{Authors' contributions}

CNM and ADT conceptualise the project. CNM undertook the main experiments of the project, including cell culturing, metabolites extraction and data analysis. CGP, WRTM and MJH performed the metabolomics and relevant data analysis. MK performed the animal infections experiments. CNM wrote the first draft and all authors contributed to completing the manuscript. All authors read and approved the final manuscript.

\section{Author details}

${ }^{1}$ Laboratory of Molecular \& Evolutionary Parasitology, RAPID Group, School of Biosciences, University of Kent, Canterbury, UK. ${ }^{2}$ Biomolecular NMR Facility, School of Biosciences, University of Kent, Canterbury, UK. ${ }^{3}$ Institute of Parasitology, Biology Centre CAS, Ceske Budejovice, Czech Republic. ${ }^{4}$ Faculty 
of Agriculture, University of South Bohemia in České Budějovice, Ceske Budejovice, Czech Republic. ${ }^{5}$ Present Address: Complex Carbohydrate Research Center, University of Georgia, Athens, GA 30602, USA. ${ }^{6}$ Present Address: School of Chemistry, University of Leeds, Leeds LS2 9JT, UK.

\section{Acknowledgements}

We thank Dr. Michelle Rowe for NMR technical support at Kent and members of the Dr. Tsaousis and Prof. Kváč laboratories for their intellectual and methodological support.

\section{Competing interests}

The authors declare that they have no competing interests.

\section{Availability of data and materials}

The data generated or analysed during the current study will be available upon request from the corresponding author.

\section{Consent for publication}

Not applicable.

\section{Ethics approval and consent to participate}

This study was carried out in accordance with Act No 246/1992 Coll. of the Czech Republic. The protocol was approved by the Committee for Animal Welfare of Biology Centre Czech Academy of Science and the veterinary administration authorities with regards to the animal experiments (experiment reference numbers: 114/2013 and 52/2014).

\section{Funding}

This research was supported by BBSRC (UK) research grant (BB/M009971/1) to Dr. Anastasios Tsaousis and a Wellcome Trust Equipment Grant 091 163/Z/10/Z (UK) to Dr. Mark J. Howard. Christopher N. Miller is supported by a GTA studentship from the School of Biosciences, University of Kent and a Research Visit Grant award from the Microbiology Society. Martin Kváč is supported by The Czech Science Foundation (Project No. 15-01090S).

\section{Publisher's Note}

Springer Nature remains neutral with regard to jurisdictional claims in published maps and institutional affiliations.

Received: 4 December 2018 Accepted: 19 March 2019 Published online: 03 April 2019

\section{References}

1. Checkley W, White AC Jr, Jaganath D, Arrowood MJ, Chalmers RM, Chen XM, Fayer R, Griffiths JK, Guerrant RL, Hedstrom L, Huston CD, Kotloff KL, Kang G, Mead JR, Miller M, Petri WA Jr, Priest JW, Roos DS, Striepen B, Thompson RC, Ward HD, Van Voorhis WA, Xiao L, Zhu G, Houpt ER. A review of the global burden, novel diagnostics, therapeutics, and vaccine targets for Cryptosporidium. Lancet Infect Dis. 2015;15(1):85-94.

2. Kotloff KL, Nataro JP, Blackwelder WC, Nasrin D, Farag TH, Panchalingam S, Wu Y, Sow SO, Sur D, Breiman RF, Faruque AS, Zaidi AK, Saha D, Alonso PL, Tamboura B, Sanogo D, Onwuchekwa U, Manna B, Ramamurthy T, Kanungo S, Ochieng JB, Omore R, Oundo JO, Hossain A, Das SK, Ahmed S, Qureshi S, Quadri F, Adegbola RA, Antonio M, Hossain MJ, Akinsola A, Mandomando I, Nhampossa T, Acacio S, Biswas K, O'Reilly CE, Mintz ED, Berkeley LY, Muhsen K, Sommerfelt H, Robins-Browne RM, Levine MM. Burden and aetiology of diarrhoeal disease in infants and young children in developing countries (the Global Enteric Multicenter Study, GEMS): a prospective, case-control study. Lancet. 2013;382(9888):209-22.

3. Striepen B. Parasitic infections: time to tackle cryptosporidiosis. Nature. 2013;503(7475):189-91.

4. Wanyiri JW, Kanyi H, Maina S, Wang DE, Steen A, Ngugi P, Kamau T, Waithera T, O'Connor R, Gachuhi K, Wamae CN, Mwamburi M, Ward HD. Cryptosporidiosis in HIV/AIDS patients in Kenya: clinical features, epidemiology, molecular characterization and antibody responses. Am J Trop Med Hyg. 2014:91(2):319-28.

5. O'Connor RM, Shaffie R, Kang G, Ward HD. Cryptosporidiosis in patients with HIV/AIDS. AIDS. 2011;25(5):549-60.
6. Caccio SM. Molecular epidemiology of human cryptosporidiosis. Parassitologia. 2005;47(2):185-92.

7. Leoni F, Amar C, Nichols G, Pedraza-Diaz S, McLauchlin J. Genetic analysis of Cryptosporidium from 2414 humans with diarrhoea in England between 1985 and 2000. J Med Microbiol. 2006:55(Pt 6):703-7.

8. Shirley DA, Moonah SN, Kotloff KL. Burden of disease from cryptosporidiosis. Curr Opin Infect Dis. 2012;25(5):555-63.

9. Wielinga PR, de Vries A, van der Goot TH, MankT, Mars MH, Kortbeek LM, van der Giessen JW. Molecular epidemiology of Cryptosporidium in humans and cattle in The Netherlands. Int J Parasitol. 2008;38(7):809-17.

10. Doumbo O, Rossignol JF, Pichard E, Traore HA, Dembele TM, Diakite M, Traore F, Diallo DA. Nitazoxanide in the treatment of cryptosporidial diarrhea and other intestinal parasitic infections associated with acquired immunodeficiency syndrome in tropical Africa. Am J Trop Med Hyg. 1997;56(6):637-9.

11. Domjahn BT, Hlavsa MC, Anderson B, Schulkin J, Leon J, Jones JL. A survey of US obstetrician-gynecologists' clinical and epidemiological knowledge of cryptosporidiosis in pregnancy. Zoonoses Public Health. 2014;61(5):356-63.

12. Hussien SM, Abdella OH, Abu-Hashim AH, Aboshiesha GA, Taha MA, El-Shemy AS, El-Bader MM. Comparative study between the effect of nitazoxanide and paromomycine in treatment of cryptosporidiosis in hospitalized children. J Egypt Soc Parasitol. 2013;43(2):463-70.

13. Manjunatha UH, Vinayak S, Zambriski JA, Chao AT, Sy T, Noble CG, Bonamy GMC, Kondreddi RR, Zou B, Gedeck P, Brooks CF, Herbert GT, Sateriale A, Tandel J, Noh S, Lakshminarayana SB, Lim SH, Goodman LB, Bodenreider C, Feng G, Zhang L, Blasco F, Wagner J, Leong FJ, Striepen B, Diagana TT. A Cryptosporidium PI(4)K inhibitor is a drug candidate for cryptosporidiosis. Nature. 2017;546(7658):376-80.

14. Sparks H, Nair G, Castellanos-Gonzalez A, White AC Jr. Treatment of Cryptosporidium: what we know, gaps, and the way forward. Curr Trop Med Rep. 2015;2(3):181-7.

15. Briggs AD, Boxall NS, Van Santen D, Chalmers RM, McCarthy ND. Approaches to the detection of very small, common, and easily missed outbreaks that together contribute substantially to human Cryptosporidium infection. Epidemiol Infect. 2014;142(9):1869-76.

16. Girouard D, Gallant J, Akiyoshi DE, Nunnari J, Tzipori S. Failure to propagate Cryptosporidium spp. in cell-free culture. J Parasitol. 2006;92(2):399-400.

17. Karanis $\mathrm{P}$, Aldeyarbi HM. Evolution of Cryptosporidium in vitro culture. Int J Parasitol. 2011;41(12):1231-42.

18. Leitch GJ, He Q. Cryptosporidiosis—an overview. J Biomed Res. 2012;25(1):1-16.

19. Muller J, Hemphill A. In vitro culture systems for the study of apicomplexan parasites in farm animals. Int J Parasitol. 2013;43(2):115-24.

20. Morada M, Lee S, Gunther-Cummins L, Weiss LM, Widmer G, Tzipori S, Yarlett N. Continuous culture of Cryptosporidium parvum using hollow fiber technology. Int J Parasitol. 2016;46(1):21-9.

21. Bones AJ, Josse L, More C, Miller CN, Michaelis M, Tsaousis AD. Past and future trends of Cryptosporidium in vitro research. Exp Parasitol. 2019;196:28-37.

22. Miller CN, Josse L, Brown I, Blakeman B, Povey J, Yiangou L, Price M, Cinatl $\mathrm{J}$, Xue WF, Michaelis M, Tsaousis AD. A cell culture platform for Cryptosporidium that enables long-term cultivation and new tools for the systematic investigation of its biology. Int J Parasitol. 2018:48(3-4):197-201.

23. Josse L, Bones AJ, Purton T, Michaelis M, Tsaousis AD. A cell culture platform for the cultivation of Cryptosporidium parvum. Curr Protoc Microbiol. 2019. https://doi.org/10.1002/cpmc.80.

24. Miller CN, Josse L, Tsaousis AD. Localization of Fe-S biosynthesis machinery in Cryptosporidium parvum mitosome. J Eukaryot Microbiol. 2018;65(6):913-22.

25. Sponseller JK, Griffiths JK, Tzipori S. The evolution of respiratory Cryptosporidiosis: evidence for transmission by inhalation. Clin Microbiol Rev. 2014:27(3):575-86.

26. Allman EL, Painter HJ, Samra J, Carrasquilla M, Llinás M. Metabolomic profiling of the malaria box reveals antimalarial target pathways. Antimicrob Agents Chemother. 2016;60(11):6635-49.

27. Ng JS, Ryan U, Trengove RD, Maker GL. Development of an untargeted metabolomics method for the analysis of human faecal samples using Cryptosporidium-infected samples. Mol Biochem Parasitol. 2012;185(2):145-50. 
28. Ng Hublin JS, Ryan U, Trengove R, Maker G. Metabolomic profiling of faecal extracts from Cryptosporidium parvum infection in experimental mouse models. PLoS ONE. 2013;8(10):e77803.

29. Saric J, Wang Y, Li J, Coen M, Utzinger J, Marchesi JR, Keiser J, Veselkov K, Lindon JC, Nicholson JK, Holmes E. Species variation in the fecal metabolome gives insight into differential gastrointestinal function. J Proteome Res. 2008;7(1):352-60

30. Tedros B, Somorjai RL, Smith IC. MR metabolomics of fecal extracts: applications in the study of bowel diseases. Magn Reson Chem. 2009;47:S54-61.

31. Hong Y, Ahn Y, Park J, Lee J, Lee H, Huh C, Kim D, Ryu DH, Hwang G. ${ }^{1} H$ NMR-based metabonomic assessment of probiotic effects in a colitis mouse model. Arch Pharm Res. 2010;33(7):1091-101.

32. Wu J, An Y, Yao J, Wang Y, Tang H. An optimised sample preparation method for NMR-based faecal metabonomic analysis. Analyst. 2010;135(5):1023-30.

33. Jacobs DM, Deltimple N, van Velzen E, van Dorsten FA, Bingham M, Vaughan EE, van Duynhoven J. ${ }^{1} H$ NMR metabolite profiling of feces as a tool to assess the impact of nutrition on the human microbiome. NMR Biomed. 2008;21(6):615-26.

34. Sengupta A, Ghosh S, Das BK, Panda A, Tripathy R, Pied S, Ravindran B, Pathak S, Sharma S, Sonawat HM. Host metabolic responses to Plasmodium falciparum infections evaluated by $1 \mathrm{H}$ NMR metabolomics. Mol BioSyst. 2016;12(11):3324-32.

35. Kostidis S. Quantitative analysis of central energy metabolism in cell culture samples. In: Giera M, editor. Clinical metabolomics: methods and protocols. New York: Springer; 2018. p. 329-42.

36. Kim TT, Parajuli N, Sung MM, Bairwa SC, Levasseur J, Soltys CM, Wishart DS, Madsen K, Schertzer JD, Dyck JRB. Fecal transplant from resveratrol-fed donors improves glycaemia and cardiovascular features of the metabolic syndrome in mice. Am J Physiol Endocrinol Metab. 2018;315(4):E511-9.

37. Xia J, Sinelnikov IV, Han B, Wishart DS. MetaboAnalyst 3.0-making metabolomics more meaningful. Nucleic Acids Res. 2015;43(W1):W251-7.

38. Kvac M, Havrdova N, Hlaskova L, Dankova T, Kandera J, Jezkova J, Vitovec J, Sak B, Ortega Y, Xiao L, Modry D, Chelladurai JR, Prantlova V, McEvoy J. Cryptosporidium proliferans n. sp. (Apicomplexa: Cryptosporidiidae): molecular and biological evidence of cryptic species within gastric cryptosporidium of mammals. PLoS ONE. 2016;11(1):e0147090.

39. Novak P, Tepes P, Fistric I, Bratos I, Gabelica V. The application of LC-NMR and LC-MS for the separation and rapid structure elucidation of an unknown impurity in 5-aminosalicylic acid. J Pharm Biomed Anal. 2006;40(5):1268-72.

40. Abrahamsen MS, Templeton TJ, Enomoto S, Abrahante JE, Zhu G, Lancto CA, Deng M, Liu C, Widmer G, Tzipori S, Buck GA, Xu P, Bankier AT, Dear $\mathrm{PH}$, Konfortov BA, Spriggs HF, Iyer L, Anantharaman V, Aravind L, Kapur V. Complete genome sequence of the apicomplexan, Cryptosporidium parvum. Science. 2004;304(5669):441-5.

41. Doyle PS, Kanaani J, Wang CC. Hypoxanthine, guanine, xanthine phosphoribosyltransferase activity in Cryptosporidium parvum. Exp Parasitol. 1998;89(1):9-15.

42. Clark DP. New insights into human cryptosporidiosis. Clin Microbiol Rev. 1999:12(4):554-63.

43. Feng H, Nie W, Sheoran A, Zhang Q, Tzipori S. Bile acids enhance invasiveness of Cryptosporidium spp. into cultured cells. Infect Immun. 2006;74(6):3342-6.

44. Gold D, Stein B, Tzipori S. The utilization of sodium taurocholate in excystation of Cryptosporidium parvum and infection of tissue culture. J Parasitol. 2001;87(5):997-1000.

45. King BJ, Keegan AR, Phillips R, Fanok S, Monis PT. Dissection of the hierarchy and synergism of the bile derived signal on Cryptosporidium parvum excystation and infectivity. Parasitology. 2012;139(12):1533-46.

46. Kar S, Daugschies A, Cakmak A, Yilmazer N, Dittmar K, Bangoura B. Cryptosporidium parvum oocyst viability and behaviour of the residual body during the excystation process. Parasitol Res. 2011;109(6):1719-23.
47. Goodgame RW, Kimball K, Ou CN, White AC Jr, Genta RM, Lifschitz $\mathrm{CH}$, Chappell $\mathrm{CL}$. Intestinal function and injury in acquired immunodeficiency syndrome-related cryptosporidiosis. Gastroenterology. 1995;108(4):1075-82

48. Kapembwa MS, Bridges C, Joseph AE, Fleming SC, Batman P, Griffin GE. Ileal and jejunal absorptive function in patients with AIDS and enterococcidial infection. J Infect. 1990;21(1):43-53.

49. Augagneur Y, Jaubert L, Schiavoni M, Pachikara N, Garg A, Usmani-Brown S, Wesolowski D, Zeller S, Ghosal A, Cornillot E, Said HM, Kumar P, Altman $\mathrm{S}$, Ben Mamoun C. Identification and functional analysis of the primary pantothenate transporter, PfPAT, of the human malaria parasite Plasmodium falciparum. J Biol Chem. 2013;288(28):20558-67.

50. Tsaousis AD, Kunji ER, Goldberg AV, Lucocq JM, Hirt RP, Embley TM. A novel route for ATP acquisition by the remnant mitochondria of Encephalitozoon cuniculi. Nature. 2008;453(7194):553-6.

51. Giris M, Depboylu B, Dogru-Abbasoglu S, Erbil Y, Olgac V, Alis H, AykacToker G, Uysal M. Effect of taurine on oxidative stress and apoptosisrelated protein expression in trinitrobenzene sulphonic acid-induced colitis. Clin Exp Immunol. 2008;152(1):102-10.

52. Green TR, Fellman JH, Eicher AL, Pratt KL. Antioxidant role and subcellular location of hypotaurine and taurine in human neutrophils. Biochim Biophys Acta. 1991;1073(1):91-7.

53. Zhang M, Izumi I, Kagamimori S, Sokejima S, Yamagami T, Liu Z, Qi B. Role of taurine supplementation to prevent exercise-induced oxidative stress in healthy young men. Amino Acids. 2004;26(2):203-7.

54. Lin S, Sanders DS, Gleeson JT, Osborne C, Messham L, Kurien M. Longterm outcomes in patients diagnosed with bile-acid diarrhoea. Eur J Gastroenterol Hepatol. 2016;28(2):240-5

55. Niggli $V$, Sigel E, Carafoli E. Inhibition of the purified and reconstituted calcium pump of erythrocytes by micro M levels of DIDS and NAP-taurine. FEBS Lett. 1982;138(2):164-6.

56. Yu H, Guo Z, Shen S, Shan W. Effects of taurine on gut microbiota and metabolism in mice. Amino Acids. 2016;48(7):1601-17.

57. Guo F, Zhang H, Payne HR, Zhu G. Differential gene expression and protein localization of Cryptosporidium parvum fatty acyl-CoA synthetase isoforms. J Eukaryot Microbiol. 2016;63(2):233-46.

58. Seeber F, Soldati-Favre D. Metabolic pathways in the apicoplast of apicomplexa. Int Rev Cell Mol Biol. 2010;281:161-228.

59. Meloni BP, Andrew Thompson RC. Simplified methods for obtaining purified oocysts from mice and for growing Cryptosporidium parvum in vitro. J Parasitol. 1996;82(5):757-62.

60. Milacek P, Vitovec J. Differential staining of Cryptosporidia by anilinecarbol-methyl violet and tartrazine in smears from faeces and scrapings of intestinal mucosa. 1985;32(1):50.

61. Morgan-Ryan UM, Fall A, Ward LA, Hijjawi N, Sulaiman I, Fayer R, Thompson RC, Olson M, Lal A, Xiao L. Cryptosporidium hominis n. sp. (Apicomplexa: Cryptosporidiidae) from Homo sapiens. J Eukaryot Microbiol. 2002;49(6):433-40.

62. Bastow EL, Peswani AR, Tarrant DSJ, Pentland DR, Chen X, Morgan A, Staniforth GL, Tullet JM, Rowe ML, Howard MJ, Tuite MF, Gourlay CW. New links between SOD1 and metabolic dysfunction from a yeast model of amyotrophic lateral sclerosis. J Cell Sci. 2016;129(21):4118.

63. Holyoake LV, Hunt S, Sanguinetti G, Cook GM, Howard MJ, Rowe ML, Poole RK, Shepherd M. CydDC-mediated reductant export in Escherichia coli controls the transcriptional wiring of energy metabolism and combats nitrosative stress. Biochem J. 2016;473(6):693-701.

64. Tarrant DJ, Stirpe M, Rowe M, Howard MJ, von der Haar T, Gourlay CW. Inappropriate expression of the translation elongation factor 1A disrupts genome stability and metabolism. J Cell Sci. 2016;129(24):4455-65.

65. Wagstaff JL, Masterton RJ, Povey JF, Smales CM, Howard MJ. (1)H NMR spectroscopy profiling of metabolic reprogramming of Chinese hamster ovary cells upon a temperature shift during culture. PLoS ONE. 2013;8(10):e77195. 